\title{
The perils of misinformation: when health literacy goes awry
}

Peter J. Schulz $\mathbb{1 D}^{1 \otimes}$ and Kent Nakamoto ${ }^{2,3}$

Growing patient empowerment and the deluge of health information and misinformation have highlighted the importance of health literacy. Unfortunately, public information and literacy programmes often fail to counter the effect of misinformation. Successfully countering misinformation presents distinctive challenges beyond patient education, and may require trust built on collaborative patient-clinician relationships.

A trusting collaborative patient-clinician relationship can help to promote accurate patient knowledge

5

'Institute of Family Medicine, Faculty of Biomedical Sciences and Faculty of Communication, Culture and Society, University of Lugano, Lugano, Switzerland.

${ }^{2}$ Virginia Polytechnic Institute and State University, Blacksburg, VA, USA.

${ }^{3}$ Faculty of Communication, Culture and Society, University of Lugano, Lugano, Switzerland.

凶e-mail:peter.schulz@usi.ch https://doi.org/10.1038/ s41581-021-00534-Z
Patient empowerment has emerged as a force in health care. It enables patients - indeed, expects them - to participate more actively in health-care decisions to improve patient-clinician relationships, patient satisfaction and health outcomes ${ }^{1}$. Active patient participation is also a natural (perhaps even unconscious) product of the availability of vast quantities of medical information on the internet and social media, directto-consumer advertising of prescription pharmaceutical drugs and direct-to-consumer marketing of medical tests. Patient empowerment also highlights the importance of health literacy as a crucial requirement to enable people to make choices that advance their health goals ${ }^{2}$. Unfortunately, as vividly reflected in the controversies over COVID-19 vaccination and treatment, seeking to improve literacy by providing accurate and adequate information can fail to ensure that patients make healthy choices. Countering mistaken beliefs must go beyond addressing gaps in patient knowledge and even well-designed communication programmes can fail. A trusting collaborative patient-clinician relationship can help to promote accurate patient knowledge, understanding and healthy choices.

Originally focused on functional literacy (the ability to read and understand medical information), health literacy has broadened to include more complex abilities, including access to information and the understanding, appraisal and application of knowledge ${ }^{3}$. Focusing on knowledge makes literacy specific to contexts (for example, diabetes literacy). The broadened vision enriches understanding of how health literacy can contribute to informed health decision-making but also reveals new ways in which literacy can fail and lead to serious health consequences, including poorer health status, and increased health costs.

We may consider, for example, diabetes, which is a major cause of chronic kidney disease. Despite the range of medical treatments that are available for this condition, many patients (perhaps a third to half of those diagnosed) forego taking medications as prescribed. Beyond economic constraints, some patients who demonstrate low adherence cite diet and exercise as preferred alternatives to medication, and claim that medications are ineffective or unsafe ${ }^{4}$. Education programmes for patients with diabetes have sought to improve patients' knowledge and understanding (literacy) of their health condition with the goal of improving patient adherence. For example, the UK Expert Patient Programme for diabetes provides patients with information filtered for accuracy and relevance, and guidance from professionals to help patients to understand their condition and its management. Participating patients report that the programme has helped them to manage their diabetes and use new skills to improve their quality of life.

\section{Misinformation: a dangerous literacy failure}

Outside of professionally managed programmes (particularly on the internet or through social media), patients might be exposed to information that is incomplete, of questionable relevance or simply wrong. Accruing such misinformation is unlikely to contribute to improved health. As literacy failures, being uninformed and being misinformed can both lead to flawed judgments and decisions that result in negative outcomes. However, these failures are substantially different and require different responses. The traditional answer to lack of information is education. As described above, literacy programmes in specific areas use tailored communications and training to improve knowledge and skills, aiming to enable choices that enhance good health outcomes.

Campaigns seeking to correct misinformation also seek to (re)educate individuals, and much recent research examines how to accomplish this goal ${ }^{5}$. Addressing misinformation not only requires imparting knowledge but also correcting mistaken beliefs; research has shown that people who are misinformed can be very persistent in their beliefs ${ }^{6}$. Persistence increases when information (accurate or not) is consistent with pre-existing beliefs or contributes to the coherence of a story or schema, 
Addressing misinformation not only requires imparting knowledge but also correcting mistaken beliefs when it is perceived to be widely shared and when the source is perceived to be credible.

If the misinformation is consistent with one's world view, it can be maintained even in the face of retraction because it is hard to disentangle the misinformation from the world view. Retraction can also create a gap in one's schema that demands to be filled. Unless the retraction also refills the gap, it may be easier to maintain the mistaken belief. Those who are misinformed may also be motivated to maintain mistaken beliefs to avoid having to admit they were wrong, and might actively reject accurate information.

Having accrued inaccurate information, one who is misinformed might feel well-informed and capable of making judgments and decisions. Moreover, this overconfidence can persist even in the face of poor task performance. The cognitive bias known as the DunningKruger effect postulates that people have inaccurate perceptions of their performance on cognitive tasks; in particular, people who perform poorly assume that their performance is typical and therefore overrate it ${ }^{7}$.

\section{Misinformed empowerment}

The central problem raised by the persistence of misinformation and ill-informed confidence is that it negates the benefits of patient empowerment ${ }^{2}$. Patients who are misinformed but are active participants might make poor decisions that are deleterious to their health. The many deaths of patients with COVID-19 who refused vaccination because of misinformation offer vivid examples. Likewise, one study of diabetes misconceptions among patients with type 2 diabetes found that $54 \%$ of patients believed that they could feel high blood glucose levels and $23 \%$ believed that diabetes medications were not needed when glucose levels were normal. These incorrect beliefs could lead to unhealthy choices ${ }^{8}$.

\section{Building trust to counter misinformation}

The experience of illness is both frightening and frustrating, not only because of the symptoms but also because it entails a loss of control. Education to improve literacy about what can be done to manage chronic noninfectious disease is important. However, for many patients, illness can also drive a search for quick and easy fixes, promised cures and explanations of why uncomfortable advice from health professionals is wrong. Beyond education, countering the attraction of misinformation is therefore crucial. Strategies that are more traditionally linked to persuasion are suggested - for example, warning of misinformation to come (inoculation), repetition of a retraction, and avoiding repetition of the misinformation while providing an alternative narrative that makes sense of the retraction'. Still, debunking misinformation is challenging and might require further reinforcement.

Health-care professionals have a particularly important role in countering misinformation. However, patient empowerment is shifting the basis of professional influence from patient acquiescence (responding to clinician beneficence) to patient trust (responding to a collaborative relationship $)^{10}$. Many of the professional and organizational arrangements of health care today are highly transactional, leading patients to take a contractual view of their relationship with health-care professionals. This view maximizes patients' sense of individual autonomy but leaves them open to misinformation. A collaborative patient-clinician relationship stands against this self-focused vision. The relationship can be professional, similar to that of teachers and students, but it must also be personal and enable the development of a sensus communis - a sense of community that builds on the idea that 'we're in this together' regardless of differing perspectives and expertise. Such a relationship supports trust and increases the credibility of correct and corrective information provided by the clinician. More broadly, this trust is central to allowing the patient to be guided by clinician expertise rather than the vagaries of the internet and social media. On this basis, health literacy can improve the capacity of the doctor-patient dyad to advance patient well-being.

1. Funnell, M. M. et al. Empowerment: an idea whose time has come in diabetes education. Diabetes Educ. 17, 37-41 (1991).

2. Schulz, P. J. \& Nakamoto, K. Health literacy and patient empowerment in health communication: the importance of separating conjoined twins. Patient Educ. Couns. 90, 4-11 (2013).

3. Sørensen, K. et al. Health literacy and public health: a systematic review and integration of definitions and models. BMC Public Health 12, 80 (2012).

4. Huang Y. M.,Pecanac, K. E. \& Shiyanbola, O. O. "Why am i not taking medications?" barriers and facilitators of diabetes medication adherence across different health literacy levels. Qual. Health Res. 30, 2331-2342 (2020).

5. Swire-Thompson, B. \& Lazer, D. Public health and online misinformation: challenges and recommendations. Annu. Rev. Public Health 41, 433-451 (2020).

6. Chan, M. S., Jones, C. R., Hall Jamieson, K. \& Albarracín, D. Debunking: a meta-analysis of the psychological efficacy of messages countering misinformation. Psychol. Sci. 28, 1531-1546 (2017).

7. Dunning, D. The Dunning-Kruger effect. on being ignorant of one's own ignorance. Adv. Exp. Soc. Psychol. 44, 247-296 (2011).

8. Mann, D. M., Ponieman, D., Leventhal, H. \& Halm, E. A. Misconceptions about diabetes and its management among low-income minorities with diabetes. Diabetes Care 32, 591-593 (2009).

9. Lewandowsky, S., Ecker, U. K. H., Seifert, C. M., Schwarz, N. \& Cook, J. Misinformation and its correction: continued influence and successful debiasing. Psychol. Sci. Public Interest 13, 106-131 (2012).

10. Bodenheimer, T., Lorig, K., Holman, H. \& Grumbach, K. Patient self-management of chronic disease in primary care. J. Am. Med. Assoc. 288, 2469-2475 (2002).

Competing interests

The authors declare no competing interests. 